\title{
TIME-REVERSED REFOCUSING OF SURFACE WATER WAVES
}

\author{
JEAN-PIERRE FOUQUE* AND ANDRÉ NACHBIN ${ }^{\dagger}$
}

\begin{abstract}
A time-reversal mirror is, roughly speaking, a device which is capable of receiving a signal in time, keeping it in memory and sending it back into the medium in the reversed direction of time. A brief mathematical review of the time-reversal theory is presented in the context of the linear shallow water equations. In particular an explicit expression is given for the refocused pulse in the simplest time-reversal case. The explicit expression for the power spectral density of the reflection process is used to construct the highpass filter which controls the refocusing process. Time-reversal numerical experiments in the (effectively) linear regime are used to validate the nonlinear shallow water code. The numerically refocused pulse is compared with the theoretical predicted shape. Further numerical experiments illustrate the robustness of the theory. In particular the time-reversal refocusing with smaller cutoff windows, the self-averaging property and finally refocusing when the nonlinear term is small but not negligible.
\end{abstract}

Key words. water waves, inhomogeneous media, asymptotic theory

AMS subject classifications. 76B07, 76B15, 35Q

1. Introduction. A time-reversal mirror is, roughly speaking, a device which is capable of receiving an acoustic signal in time, keeping it in memory and sending it back into the medium in the reversed direction of time. In the context of ultrasounds time-reversal mirrors have been developed and their effect studied experimentally by Mathias Fink and his collaborators at the Laboratoire Ondes et Acoustique (ESPCIParis). The main effect is the refocusing of the scattered signal after time-reversal in a random medium: an acoustic pulse is sent in a disordered medium generating a "noisy" reflected signal which is time-reversed and sent back into the medium. The new reflected signal is a pure pulse with a shape similar to the initial pulse. Amazingly its "refocusing" takes place in time and space and seems to be independent of the realization of the medium, in the regime where its correlation length is smaller than the typical wavelength of the pulse. Experiments in the context of underwater acoustics have been conducted by Kuperman et al. and show the same phenomenon. We refer to reference [12] for a description of these experiments and further references.

From the theoretical point of view, a first proof of this refocusing effect has been obtained by Clouet and Fouque [10] in the context of a one-dimensional random medium for which only the time refocusing is relevant. The refocusing is obtained by using asymptotics in the regime where there are three well separated scales:

$$
\frac{\text { correlation length of the medium }}{\text { wavelength of the pulse }} \approx \frac{\text { wavelength of the pulse }}{\text { distance of propagation }} \ll 1 \text {. }
$$

The fluctuations of the medium are not assumed to be small but rather of the order of several tens of percent. This result has been extended to the case of 3D layered media by Fouque and Ndzie [16] where it is shown that refocusing takes place in time as well as in space. More recently space refocusing has been analysed in the context of small fluctuation and the parabolic approximation by Blomgren, Papanicolaou and Zhao [3]

\footnotetext{
*Department of Mathematics, North Carolina State University, Raleigh, NC 27695-8205, USA (fouque@math.ncsu.edu). This work was supported by ONR under Grant N00014-02-1-0089.

$\dagger$ Instituto de Matemática Pura e Aplicada, Est. D. Castorina 110, Jardim Botânico, Rio de Janeiro, RJ 22460-320, Brazil (nachbin@impa.br). This work was supported by CNPq/Brazil under Grant 300368/96-8 and in part by ONR under Grant N00014-02-1-0089 through this author's visit to NCSU.
} 
and Papanicolaou, Ryhzik and Sølna [23]. They show that time-reversal and randomness helps in "beating" the diffraction limit: they call this effect super-resolution. A complete proof of super-resolution for three-dimensional randomly layered media has been obtained in Fouque and Sølna [15]. Potential applications of these effects are numerous in imaging and wireless communication.

The goal of this paper is to present the time-reversal and refocusing of water waves and, of particular interest, to initiate a study of the effect of nonlinearity on the refocusing effect. For doing so we use the context of gravity driven water waves, propagating in shallow one-dimensional channels in the regime where the fluid is considered to be inviscid and incompressible. The randomness comes from the topography at the bottom of the shallow channel. Note that the shallow water regime is dictated by having a small depth to wavelength ratio. This is the same as the long wave regime. Velocity and elevation at the free surface are described through the shallow water equations which contain a Burgers' type nonlinearity controlled by the amplitude of the wave. For small amplitude-to-depth ratios the nonlinearity is negligible and we are back to the one-dimensional case studied from Clouet and Fouque [10]. Moreover a very efficient numerical code has been developed for solving these nonlinear equations. It provides an extremely accurate tool to conduct numerical experiments on the effect of nonlinearity on time-reversal refocusing. We first validate this tool in the linear regime and show, as predicted by the theory, that refocusing indeed takes place: a right travelling pulse shaped wave is sent from the left side of the random portion of the medium. It interacts with the fluctuating bottom and produces a reflected "noisy" wave propagating towards the left of the disordered channel. At the left end of the channel this wave is time-reversed by simply changing the sign of its velocity profile and sent back into the random medium. It then produces a new reflected wave which appears to be a "clean" pulse with a shape independent of the realization of the medium. The pulse shape is of a deterministic character. This is called the stabilization of the refocused pulse shape and is due to the self-averaging property. The theory provides an analytical description of this "new" (refocused) pulse which is perfectly captured by the numerical experiments.

We then increase the amplitude of the incoming pulse in order to study computationally the effect of nonlinearity. For small nonlinearity, and way before shocks form, our numerical results show that refocusing still takes place providing evidence of the robustness of this effect.

Regarding applications in water waves, the problem of waveform inversion has been studied by adjoint methods in Pires and Miranda [24] and the references within. Their goal is to characterize the initial sea surface displacement due to tsunamigenic earthquakes. In other words one would like to recover (numerically) relevant details of a tsunami source from tidal gauge observations. In our case, instead of performing the backward numerical integration of the corresponding adjoint equations, we use the (same) forward numerical model but with the time-reversed data. Waveform inversion is obtained through the time-reversed refocusing effect. In a separate work, Fouque, Garnier and Nachbin [14], the mathematical theory for the time-reversal of linear hyperbolic waves is extended to the more realistic case of dispersive waves.

This paper is organized as follows. In section 2 we present a brief review of the linear time-reversal acoustic theory in the context of the shallow water equations. In particular we give an explicit expression for the refocused pulse in the simplest timereversal case: a random medium without a slowly varying background, where timereversal is performed using the indicator function of the full time-recording interval. In 
this case we use the explicit expression for the power spectral density of the reflection process and construct the highpass filter which controls the refocusing process. In section 3 we present the nonlinear shallow water equations used in our numerical experiments. Time-reversal experiments in the (effectively) linear regime are used to validate the numerical code. In particular we compare the observed refocused pulse with its predicted shape. We also perform numerical experiments with a smaller cutoff window, we verify numerically the self-averaging property and finally we (still) observe refocusing when the nonlinear term is small but not negligible.

2. One-Dimensional Time-Reversal: A Brief Review of the Theory. In this section we briefly review the theory presented in [10]. We mainly focus on what is happening at the interface $\{x=0\}$ between the constant medium on the left and the random medium on the right (c.f. Figure 4.1). It is natural to look at the quantities of interest in time at the interface and we know that this is equivalent to look at them in space on the left half-space due to the constant speed of propagation. Also particular attention is paid to the scaling of the problem.

2.1. The Model. Let $\psi(x, t)$ be the flux function and $\eta(x, t)$ the free surface elevation. In the linear regime the shallow water equations become

$$
\left\{\begin{array}{cc}
\frac{1}{c^{2}(x)} \psi_{t} & +\eta_{x}=0 \\
\eta_{t} & +\psi_{x}=0
\end{array}\right.
$$

which are the acoustic equations where $\psi$ is the velocity of the wave and $\eta$ the pressure. Eventhough in our model the fluctuations occur naturally on the depth $h(x)=g^{-1} c^{2}(x)$, we shall rather consider them on $1 / c^{2}(x)$ which is the quantity which is naturally averaged by the wave in the homogenization process. We will explain at the end of this section how to modify the theory in order to take this into account. Our model is

$$
\frac{1}{c^{2}(x)}=\frac{1}{c_{0}^{2}}(1+\mu(x / \varepsilon))
$$

on the half-space $\{x>0\}$ with a constant speed $c_{0}$ on the half-space $\{x<0\}$. The stochastic process $\mu$ is stationary, centered, bounded $(|\mu| \leq C<1$, for some constant $C)$ and is assumed to be exponentially mixing in the sense that it "decorrelates" exponentially fast. Our examples constructed with i.i.d. random variables will satisfy this mixing condition. By rescaling $\mu$ as $\mu(x / \varepsilon)$ we make the correlation length of order $\varepsilon$ where $\varepsilon>0$ is a small parameter. Observe that the size of the fluctuations, namely $\sqrt{\left\langle\mu(0)^{2}\right\rangle}$ (where the brakets denote an average with respect to the invariant measure of $\mu$ ), is not small but rather of order a few tens of percents in our applications. The boundary conditions will correspond to a pulse incoming from the left and entering the random medium at $\{x=0\}$, and a radiation condition at $+\infty$. This last condition is achieved by making the medium constant far away to the right and imposing no left-going wave on this part of the medium. The hyperbolicity of the problem insures that at a finite time this latter boundary condition does not affect the quantities of interest on the left half-space. Observe also that, at the interface $\{x=0\}$, we have matched the constant medium on the left with the averaged medium on the right in the sense that

$$
\frac{1}{c_{0}^{2}}=\left\langle\frac{1}{c^{2}(x)}\right\rangle .
$$


In the constant left half-space we decompose the wave into the right-going wave $A=c_{0}^{1 / 2} \eta+c_{0}^{-1 / 2} \psi$, and the left-going wave $B=-c_{0}^{1 / 2} \eta+c_{0}^{-1 / 2} \psi$. Our incoming pulse at $\{x=0\}$ is given by:

$$
A(t, 0)=a f(t)
$$

where $f$ is the pulse shape (typically the derivative of a Gaussian) and $a$ represents the amplitude. This setup means that a right-going wave $A(t, x)=a f\left(t-x / c_{0}\right)$ is coming from the left $(x<0)$. The reflected signal at the interface is denoted by $B_{0}(t)$ so that it corresponds to a left-going wave $B(t, x)=B_{0}\left(t+x / c_{0}\right)$ on the left half-space.

2.2. Scales. The size of the pulse is of order one in the sense that it is independent of the small parameter $\varepsilon$ which represents the correlation length of the random medium. If we observe the reflected wave after a time of order one we will only see the result of homogenization since the wavelength is much larger than the size of the inhomogeneities. Since the homogenized medium on the right half-space matches the constant medium on the left half-space we essentially would see nothing reflected. One has to wait longer to see the fluctuations "build-up" and create a "noisy" reflected wave. The asymptotic theory is extremely precise, by using diffusion-approximation results, when the reflected wave is observed for a long time of order $1 / \varepsilon$. In this case we are in the white noise regime inside of the medium and the powerfull tools of stochastic calculus associated to Brownian motion can be used (we refer to [8] for more details).

Since we are dealing with long times of order $1 / \varepsilon$ and therefore long distances of propagation of the same order, it is convenient to rescale the problem by setting $t=t^{\prime} / \varepsilon$ and $x=x^{\prime} / \varepsilon$ so that $x^{\prime}$ and $t^{\prime}$ become our macroscopic space and time variables. The correlation length becomes $\varepsilon^{2}$ since $\mu(x / \varepsilon)$ becomes $\mu\left(x^{\prime} / \varepsilon^{2}\right)$ and the typical wavelength becomes $\varepsilon$ since the pulse is now $f\left(t^{\prime} / \varepsilon\right)$. The speed $c_{0}$ of the uniform medium does not change in this macroscopic scale.

2.3. Integral representation. Using Fourier transform in time one can derive the following integral representation for the reflected wave $[1,10]$ :

$$
B_{0}\left(t^{\prime}\right)=\frac{a}{2 \pi} \int e^{-i \omega \frac{t^{\prime}}{\varepsilon}} \hat{f}(\omega) R^{\varepsilon}(0, \omega) d \omega
$$

where $R^{\varepsilon}(0, \omega)$ is the reflection coefficient of the random medium at the interface $x=0$, when, at frequency $\omega$, a wave of unit amplitude is incoming from the left.

2.4. Time-Reversal. Time-reversal consists in using the reflected wave (or a part of it) as a new incoming wave in the reversed direction of time. We send the initial pulse and then monitor the reflected wave up to time $t_{0}^{\prime}$. Let us denote by $G_{t_{0}^{\prime}}\left(t^{\prime}\right)$ a cutoff function with support in $\left[0, t_{0}^{\prime}\right]$. The piece of reflected wave is

$$
B_{0}\left(t^{\prime}\right) G_{t_{0}^{\prime}}\left(t^{\prime}\right)
$$

which after time-reversal becomes $B_{0}\left(t_{0}^{\prime}-t^{\prime}\right) G_{t_{0}^{\prime}}\left(t_{0}^{\prime}-t^{\prime}\right)$. In order to use the integral representation formula (2.2) one needs to compute the Fourier transform of this new incoming wave in the original time scale $t=t^{\prime} / \varepsilon$. We set

$$
g(t)=B_{0}\left(t_{0}^{\prime}-\varepsilon t\right) G_{t_{0}^{\prime}}\left(t_{0}^{\prime}-\varepsilon t\right)
$$


and we compute its Fourier transform:

$$
\hat{g}(\omega)=\frac{a}{2 \pi \varepsilon} e^{i \omega \frac{t_{0}^{\prime}}{\varepsilon}} \int \hat{\hat{f}}\left(\omega^{\prime}\right) \overline{R^{\varepsilon}\left(0, \omega^{\prime}\right)} \overline{G_{t_{0}^{\prime}}}\left(\frac{\omega-\omega^{\prime}}{\varepsilon}\right) d \omega^{\prime}
$$

where, for simplicity, we have dropped the $t_{0}^{\prime}$-subscript in $G$. Sending back into the random medium the time-reversed wave $g(t)$ will produce a new reflected wave denoted by $B_{0}^{T R}\left(t^{\prime}\right)$. Looking at it after a long time and in the scale of the original pulse means that we set $t^{\prime}=t_{1}^{\prime}+\varepsilon t$ and using the integral representation formula (2.2) we get:

$$
B_{0}^{T R}\left(t_{1}^{\prime}+\varepsilon t\right)=\frac{1}{(2 \pi)} \int e^{-i \omega t} e^{-i \omega \frac{t_{1}^{\prime}}{\varepsilon}} \hat{g}(\omega) R^{\varepsilon}(0, \omega) d \omega .
$$

Combined with (2.3) we get that $B_{0}^{T R}$ is given as a double frequency integral:

$$
\begin{aligned}
& B_{0}^{T R}\left(t_{1}^{\prime}+\varepsilon t\right)= \\
& \frac{a}{(2 \pi)^{2} \varepsilon} \iint e^{-i \omega t} e^{-i \omega\left(\frac{t_{1}^{\prime}-t_{0}^{\prime}}{\varepsilon}\right)} \overline{\hat{f}}\left(\omega^{\prime}\right) R^{\varepsilon}(0, \omega) \overline{R^{\varepsilon}\left(0, \omega^{\prime}\right)} \overline{\hat{G}_{t_{0}^{\prime}}}\left(\frac{\omega-\omega^{\prime}}{\varepsilon}\right) d \omega^{\prime} d \omega .
\end{aligned}
$$

After the change of variables $\omega^{\prime}=\omega+\varepsilon h$, it becomes:

$$
\begin{aligned}
& B_{0}^{T R}\left(t_{1}^{\prime}+\varepsilon t\right)= \\
& \frac{a}{(2 \pi)^{2}} \int e^{-i \omega t}\left(\int \overline{\hat{f}}(\omega+\varepsilon h) R^{\varepsilon}(0, \omega) \overline{R^{\varepsilon}(0, \omega+\varepsilon h)} \overline{G_{t_{0}^{\prime}}}(-h) d h\right) e^{-i \omega\left(\frac{t_{1}^{\prime}-t_{0}^{\prime}}{\varepsilon}\right)} d \omega .
\end{aligned}
$$

2.5. Asymptotics. Looking first at the expected reflected wave consists in taking the expectation of the previous integral representation of $B_{0}^{T R}\left(t_{1}^{\prime}+\varepsilon t\right)$, in other words, by averaging over many realizations of the medium. By linearity of the integral we get:

$$
\begin{aligned}
& B_{0}^{T R}\left(t_{1}^{\prime}+\varepsilon t\right)= \\
& \frac{a}{(2 \pi)^{2}} \int e^{-i \omega t}\left(\int \hat{\hat{f}}(\omega+\varepsilon h) \mathbb{E}\left\{R^{\varepsilon}(0, \omega) \overline{R^{\varepsilon}(0, \omega+\varepsilon h)}\right\} \overline{G_{t_{0}^{\prime}}}(-h) d h\right) e^{-i \omega\left(\frac{t_{1}^{\prime}-t_{0}^{\prime}}{\varepsilon}\right)} d \omega .
\end{aligned}
$$

The asymptotic analysis of the wave equations inside the random medium shows that $\mathbb{E}\left\{R^{\varepsilon}(0, \omega) \overline{R^{\varepsilon}(0, \omega+\varepsilon h)}\right\}$ converges, as $\varepsilon$ goes to zero, to a quantity that we shall denote by $u(\omega, h)$. On the other hand $\overline{\hat{f}}(\omega+\varepsilon h)$ converges to $\overline{\hat{f}}(\omega)$ and this integral goes to zero due to the fast phase $\exp \left(-i \omega\left(t_{1}^{\prime}-t_{0}^{\prime}\right) / \varepsilon\right)$, unless $t_{1}^{\prime}=t_{0}^{\prime}$. In that case the limit is simply:

$$
\lim _{\varepsilon \rightarrow 0} \mathbb{E}\left\{B_{0}^{T R}\left(t_{0}^{\prime}+\varepsilon t\right)\right\}=\frac{a}{(2 \pi)^{2}} \int e^{-i \omega t} \overline{\hat{f}}(\omega)\left(\int u(\omega, h) \overline{G_{t_{0}^{\prime}}}(-h) d h\right) d \omega .
$$

Introducing the Fourier transform $\Lambda\left(\omega, t^{\prime}\right)$ of $u(\omega, h)$, we get:

$$
\lim _{\varepsilon \rightarrow 0} \mathbb{E}\left\{B_{0}^{T R}\left(t_{0}^{\prime}+\varepsilon t\right)\right\}=\frac{a}{2 \pi} \int e^{-i \omega t} \overline{\hat{f}}(\omega)\left(\Lambda(\omega, \cdot) \star G_{t_{0}^{\prime}}(-\cdot)\right)(0) d \omega
$$


which, as a function of $t$ is the initial pulse $a f(t)$ convolved with $H_{t_{0}^{\prime}}(t)$ where $H_{t_{0}^{\prime}}$ denotes the inverse Fourier transform of $\left(\Lambda(\omega, \cdot) \star G_{t_{0}^{\prime}}(-\cdot)\right)(0)$ with respect to $\omega$ :

$$
\lim _{\varepsilon \rightarrow 0} \mathbb{E}\left\{B_{0}^{T R}\left(t_{0}^{\prime}+\varepsilon t\right)\right\}=\left(a f \star H_{t_{0}^{\prime}}\right)(t) .
$$

This convergence is actually much stronger and the limit is in fact the same for all realizations (except for a set of realizations of measure zero). This is obtained by performing the asymptotic analysis of $\mathbb{E}\left\{B_{0}^{T R}\left(t_{0}^{\prime}+\varepsilon t\right)^{2}\right\}$ which involves four frequencies:

$$
\mathbb{E}\left\{R^{\varepsilon}\left(0, \omega_{1}\right) \overline{R^{\varepsilon}\left(0, \omega_{1}+\varepsilon h_{1}\right)} R^{\varepsilon}\left(0, \omega_{2}\right) \overline{R^{\varepsilon}\left(0, \omega_{2}+\varepsilon h_{2}\right)}\right\} .
$$

It can be shown (for instance in [5]) that, in the limit $\varepsilon \downarrow 0$, the $\left(\omega_{1}, h_{1}\right)$-terms decorrelate from the $\left(\omega_{2}, h_{2}\right)$-terms and one can deduce that the variance of $B_{0}^{T R}\left(t_{0}^{\prime}+\right.$ $\varepsilon t)$ goes to zero. The time refocusing property can be summarized in:

$$
\lim _{\varepsilon \rightarrow 0} B_{0}^{T R}\left(t_{1}^{\prime}+\varepsilon t\right)=\left\{\begin{array}{ccc}
0 & \text { if } & t_{1}^{\prime} \neq t_{0}^{\prime} \\
\left(a f \star H_{t_{0}^{\prime}}\right)(t) & \text { if } & t_{1}^{\prime}=t_{0}^{\prime}
\end{array}\right.
$$

In the homogenous medium case (constant background) we have considered here, $\Lambda(\omega, t)$ can be computed explicitly [4]:

$$
\Lambda(\omega, t)=\frac{\alpha_{\mu} \omega^{2}}{\left(1+\omega^{2} \alpha_{\mu} t\right)^{2}}
$$

where

$$
\alpha_{\mu}=\int_{0}^{\infty} \mathbb{E}\{\mu(0) \mu(x) d x\}
$$

is the integrated correlation of the medium. Observe that taking $t_{0}^{\prime}$ large and $G$ the indicator function of $\left[0, t_{0}^{\prime}\right]$ means that we are sending back the full reflected wave. In this case $\left(\Lambda(\omega, \cdot) \star G_{t_{0}^{\prime}}(-\cdot)\right)(0)$ can be computed explicitly since

$$
\left(\Lambda(\omega, \cdot) \star G_{t_{0}^{\prime}}(-\cdot)\right)(0)=\int_{0}^{\infty} \Lambda(\omega, \tau) G_{t_{0}^{\prime}}(\tau-t) d \tau=\int_{0}^{t_{0}^{\prime}} \frac{\alpha_{\mu} \omega^{2}}{\left(1+\alpha_{\mu} \omega^{2} \tau\right)^{2}} d \tau=\frac{\alpha_{\mu} \omega^{2} t_{0}^{\prime}}{1+\alpha_{\mu} \omega^{2} t_{0}^{\prime}} .
$$

This can be interpreted as a highpass filter in the expression where the time-reversed pulse is given by

$$
f^{T R}(t)=\frac{a}{2 \pi} \int e^{-i \omega t} \overline{\hat{f}}(\omega)\left(\frac{\alpha_{\mu} \omega^{2} t_{0}^{\prime}}{1+\alpha_{\mu} \omega^{2} t_{0}^{\prime}}\right) d \omega
$$

The filter for the time-reversal procedure has been graphed in Figure 2.1. Note that this highpass filter becomes identically one in the limit $t_{0}^{\prime} \rightarrow \infty$. Figure 2.1 confirms this fact. Therefore $H_{t_{0}^{\prime}}$ tends to a delta function and the initial pulse af is exactly recovered through time reversal.

2.6. Almost Stationary Case. In the context of this paper, shallow water surface waves, it is natural to model the fluctuations of the bottom of the channel or equivalently the depth of the channel. We do that by putting random depths at the mesh points $j \varepsilon$, on a grid of mesh $\varepsilon$, inside the random slab:

$$
h(j \varepsilon)=h_{0}\left(1+\sigma Z_{j}\right)
$$



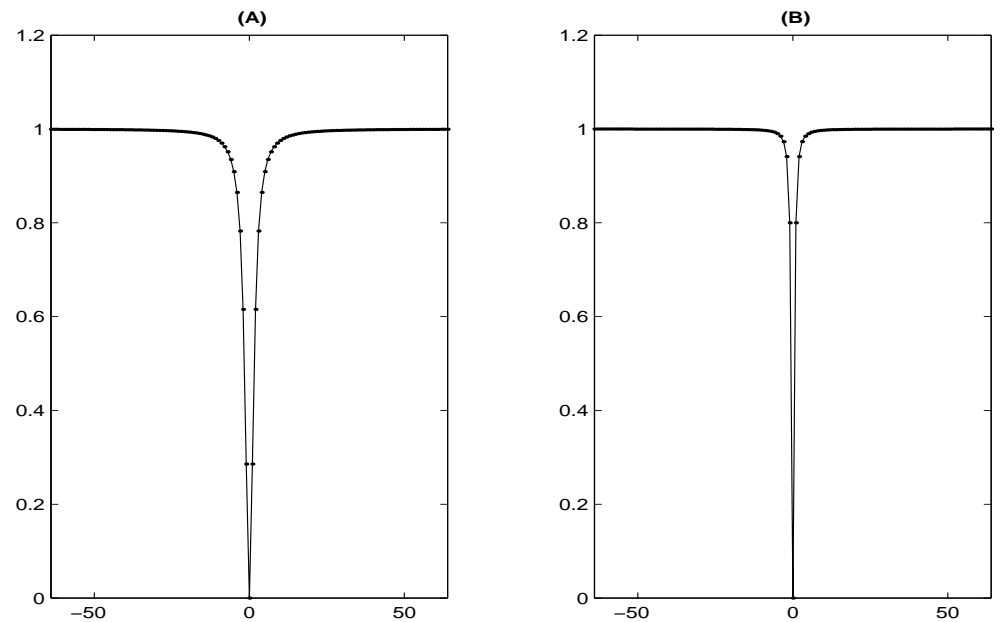

FIG. 2.1. The right and left graphs illustrate the highpass filter for the time-reversed reflection process. Graph (A) describes the filter used to obtain the theoretical pulse shape to be presented in Figure 4.4, namely for $t_{0}^{\prime}=0.5$. In graph $(\mathrm{B})$ we let $t_{0}^{\prime}=5$. Note that the time-reversed refocused signal will contain (nearly) all the Fourier content of the initial data. In both cases we used $\alpha_{\mu}=0.08$. This value is calculated below.

where the random variables $Z_{j}$ 's are independent and identically uniformly distributed over $(-1,1)$ for instance, while $0<\sigma<1$ "measure" the size of the fluctuations (several tens of percent typically). We then define $h(x)$ by an interpolation procedure. Here we chose the linear interpolation because it is very convenient for the efficiency of the numerical code. In fact the nature of the results explained in Section 2.5 does not depend on that particular choice but only the parameters $c_{0}$ and $\alpha$ are affected. The model for $1 / c^{2}(x)$ is then given by

$$
\frac{1}{c^{2}(x)}=\frac{1}{g h(x)}=\frac{1}{\left.g h_{0}(1+\mu(x / \varepsilon))\right)}
$$

where

$$
\mu(x / \varepsilon)=\sigma Z_{j}+\sigma\left(\frac{x}{\varepsilon}-j\right)\left(Z_{j+1}-Z_{j}\right)
$$

for $j \varepsilon \leq x<(j+1) \varepsilon$. This model, compared to the one in Section 2.1, poses two problems:

1. The homogenized medium is not $1 / g h_{0}$.

2. The stochastic process $\mu$ is not stationary.

The correct homogenized medium is obtained by:

$$
\frac{1}{\bar{c}^{2}}=\lim _{L \rightarrow \infty} \frac{1}{L} \int_{0}^{L} \frac{1}{g h_{0}(1+\mu(y))} d y
$$

which is also given by:

$$
\frac{1}{\bar{c}^{2}}=\mathbb{E}\left\{\int_{j}^{j+1} \frac{1}{g h_{0}\left(1+\sigma Z_{j}+\sigma(y-j)\left(Z_{j+1}-Z_{j}\right)\right)} d y\right\}
$$




$$
=\frac{1}{g h_{0}} \mathbb{E}\left\{\frac{\log \left(\frac{1+\sigma Z_{j+1}}{1+\sigma Z_{j}}\right)}{\sigma\left(Z_{j+1}-Z_{j}\right)}\right\}
$$

which can be computed by using the uniform distribution of $\left(Z_{j}, Z_{j+1}\right)$ over the square $(-1,1)^{2}$ :

$$
\frac{1}{\bar{c}^{2}}=\frac{1}{g h_{0}} \int_{-1}^{1} \int_{-1}^{1} \frac{\log \left(\frac{1+\sigma z_{j+1}}{1+\sigma z_{j}}\right)}{\sigma\left(z_{j+1}-z_{j}\right)} d z_{j} d z_{j+1}
$$

The nonstationarity of $\mu$ can be easily incorporated in the theory by replacing $\alpha_{\mu}=$ $\int_{0}^{\infty} \mathbb{E}\{\mu(0) \mu(x)\} d x$ by the modified

$$
\alpha_{\mu}=\int_{0}^{\infty}\left(\lim _{T \rightarrow \infty} \frac{1}{T} \int_{0}^{T} \mathbb{E}\{\mu(y) \mu(y+x)\} d y\right) d x
$$

By using the piecewise linear random topography from equation (2.6) one can deduce

$$
\alpha_{\mu}=\frac{2}{9} \sigma^{2}
$$

For $60 \%$ bottom fluctuations (i.e. $\sigma=0.6$ ) we have that $\alpha_{\mu}=0.08$. Recall that $Z_{j}$ are iid random variables uniformly distributed over $(-1,1)$.

3. Numerical Setup. In water wave problems [26] the following characteristic scales are relevant: the typical depth $h_{0}$, typical wavelength $\lambda$, typical amplitude $a$ of the free surface elevation $\eta(x, t)$, the horizontal length scale $l_{b}$ of the bottom irregularities and $\mathrm{L}$ the total length of the rough region. When using dimensionless variables the following dimensionless parameters appear: $\alpha=a / h_{o}$ which controls the strength of the nonlinearity, $\beta=h_{0}^{2} / \lambda^{2}$ which controls dispersion and $\gamma=l_{b} / \lambda$ which controls how rapidly the bottom irregularities vary. These parameters arise typically in nonlinear potential theory and in particular $\alpha$ will be used to set the appropriate regime through the initial data of our computational experiments.

The nonlinear shallow water model used in our experiments is given by

$$
\begin{gathered}
U_{t}+U U_{x}+V U_{y}=-g \eta_{x} \\
V_{t}+U V_{x}+V V_{y}=-g \eta_{y} \\
\eta_{t}+[H U]_{x}+[H V]_{y}=0
\end{gathered}
$$

where $H(x, y, t)=\eta(x, y, t)+h(x, y)$. The topography is described by $-h(x, y)$. The numerical experiments are all one-dimensional. Therefore during the entire dynamics in the horizontal plane the velocity $V(x, y, t)$ will be automatically set to zero and $U$ will be $y$-independent.

Note that the linear one-dimensional shallow water system is

$$
\begin{gathered}
U_{t}+g \eta_{x}=0, \\
\eta_{t}+(h U)_{x}=0 .
\end{gathered}
$$




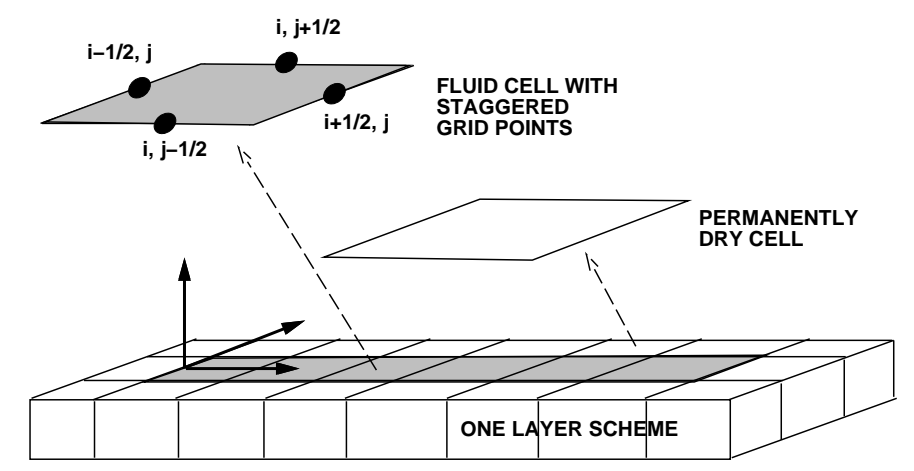

FIG. 3.1. Schematic picuture for the horizontal $2 D$ mesh. The gray area represents the fluid body while the white area the impermeable boundary.

which is analogous to the acoustic wave system [10]. It can be put in the form

$$
\begin{gathered}
\psi_{t}+(g h) \eta_{x}=0 \\
\eta_{t}+\psi_{x}=0
\end{gathered}
$$

The flux function per unit width is given by $\psi(x, t) \equiv h(x) \cdot U(x, t)$. This is the form used to describe the theory in the previous sections.

In our first experiments we will solve the nonlinear shallow water system (3.13.3 ) in the (effectively) linear regime. Linear propagation regimes are obtained by setting the amplitude-to-depth ratio, of the initial data, to be small. From previous experiments performed in Nachbin and Casulli [20] we know that $\alpha=0.001$ generates a linear wave whereas $\alpha=0.1$ produces a wave that breaks after a small time interval. To normalize the reference shallow water speed we choose our parameters so that it is equal to one in the flat regions: namely we set $g=1 / h_{0}$ so that $\left(g h_{0}\right)=1$. Several validation experiments were presented in Nachbin and Casulli [20] and the numerical solution exhibited very good conservation properties over large propagation distances.

In the numerical experiments we consider a one-dimensional channel (c.f. Figure 3.1) having a disordered topography as shown in Figure 4.1 where the vertical scale has been exagerated for graphical reasons. The influence of the topography is felt through the depth function $H(x, y, t)$. The disordered medium is constructed by sampling 5300 random heights which are allocated at the mesh points. The finite difference spacing will coincide with the small parameter $\varepsilon$ from the time-reversal problem. These random heights are then connected by straight lines to form the synthetised topography. The channel has a total length of 107 units while the disordered topography covers 53 units of length. It is allocated from node 5400 up to node 10700 (c.f. Figure 4.1). In all experiments to be presented below we use the same mesh: $\Delta x=0.01=\varepsilon, \Delta y=0.1$ and $\Delta t=0.0025$. The fluctuations are $60 \%$ of the total (unit) depth. The initial pulse is given by the derivative of a Gaussian

$$
\eta(x, 0)=U(x, 0)=-10 a\left(x-x_{j}\right) \exp \left(\frac{-\left(x-x_{j}\right)^{2}}{0.08}\right)
$$

and is centered at node $\mathrm{j}=5250$. Its effective width is equal to one. We set $\alpha=0.001$ by choosing the initial data with $a=0.001$. The topography (as in Figure 4.1) has 
been rescaled so that $h_{0}=1$. We allow the simulation to run over 52.5 units of time (21000 time steps) for which we record the reflected signal at the left end of the channel.

The equations (3.1-3.3) are discretized by an implicit, semi-Lagrangian technique which accomplishes the objective that the stability of the scheme does not depend on the celerity. A fixed staggered grid is defined on the horizontal plane. For the present one-dimensional experiments we have three lines of grid points along the horizontal direction, of which only the middle one is relevant (c.f. Figure 3.1). It runs through the middle of the one-dimensional channel. The other two are used for boundary conditions. By a semi-Lagrangian technique we mean that the numerical transport is performed in an Eulerian-Lagrangian manner, relying therefore on the interpolation of the respective grid point-values at the backward-characteristic points of departure. Details are given in Casulli and Cheng [6] and the references within.

4. Time Reversal Experiments. In this section we describe how the forward and time-reversed experiments are performed. We start by describing the geometry and scales associated to the time-reversal experiments.

4.1. Random medium and scales. First we do a forward numerical experiment with the initial pulse located to the left of the random medium as in Figure 4.1. As the experiment takes place the pulse will propagate to the right with unit speed and will interact with the disordered topography. As mentioned above the topography is synthetised by using a random number generator. Uniformly distributed heights are place at each mesh point and connected by straight lines as indicated by the (bottom) detail of Figure 4.1. The correlation length of the topography is $\varepsilon=\Delta x=0.01$, the pulse width is approximately one and the total propagation distance is equal to 50 units. With these values we are in the correct self-averaging regime indicated by the theory. We should recall that for graphical reasons the wave amplitude has been exagerated in Figure 4.1. The amplitude-to-depth ratio will be indicated in the following subsections.

4.2. Reflected signal. As mentioned above we do a forward numerical experiment with the initial pulse located to the left of the random medium as in Figure 4.1. At the end of 50 time-units the free surface disturbance is presented in the lower graph of Figure 4.2. Recall that the disordered medium is contained in $[0,53]$. Over this interval we see the transmitted pulse and behind it a wave that is undergoing multiple-scattering. Over the [-50,0] interval the channel is flat and there the wave is a left-going reflected wave. The reflected wave is recorded as shown by the top (windowed) signal in Figure 4.2. The final time is $t_{0}^{\prime} / \varepsilon=50$ and the cutoff function $G_{t_{0}^{\prime}}$ is the indicator function for the corresponding time interval $\left[0, t_{0}^{\prime}\right]$. This windowed reflected wave is time-reversed by changing the sign of the velocity profile $U(x, t)$.

\subsection{Refocusing experiments.}

4.3.1. Large window experiment. We call this a large window experiment because we are using the whole time interval for which we recorded the reflected wave. The cutoff function's support is as large as it can be for this experiment. The top graph of Figure 4.3 presents the initial condition for the time-reversal refocussing experiment. It is the same time-reversed reflected wave mentioned above. The timereversed wave propagates to the right and starts interacting with the random medium, as seen in the two intermediate graphs. At time $t=t_{0}^{\prime} / \varepsilon=50$ refocusing takes place. In the limit $\varepsilon \rightarrow 0$ we should get a perfect refocusing of the wave, with no noise around 

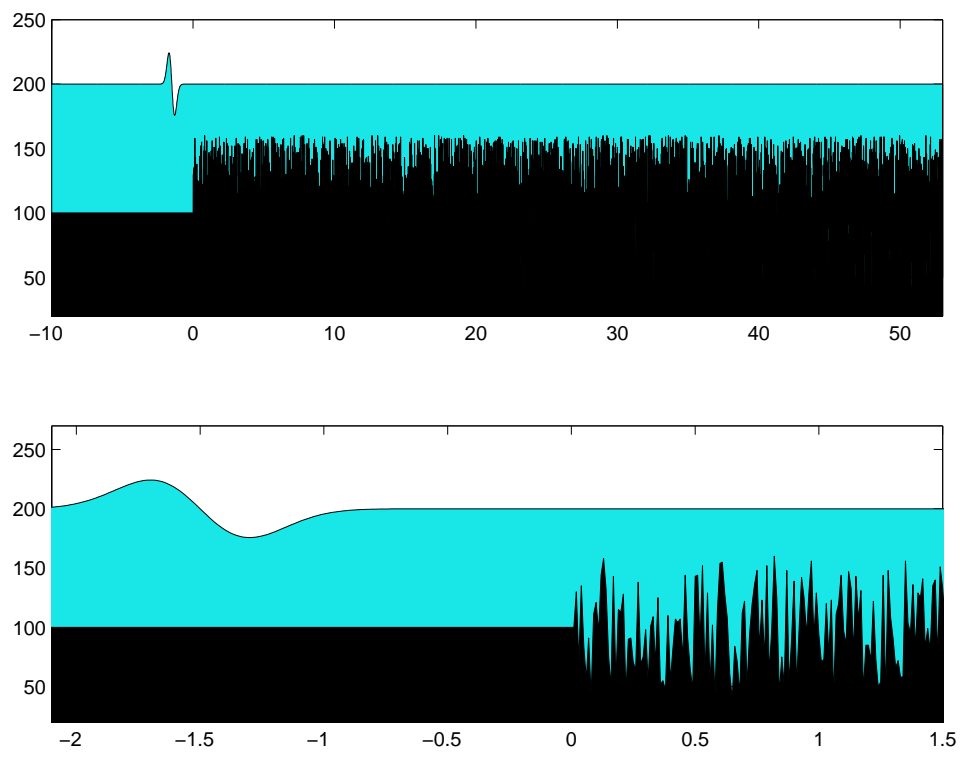

FIG. 4.1. Shallow channel with a disordered topographic right half-space. The correlation length of the bottom is 0.01 and the fluctuation level is $60 \%$ of the average depth. A pulse of unit width is incoming from the left. Its amplitude has been exagerated for graphical reason. The top picture is on the scale of typical propagation distances used in the experiments. The bottom picture is on the scale of the pulse width. Observe that pulse is large compared to the correlation length of the medium.

it. Note that for a finite value $t_{0}^{\prime} / \varepsilon=50$ we already get a very sharp refocusing of our original pulse with very little noisy to its left. This is good indication that the numerics captures the appropriate regime established by the theory. In Figure 4.4 refocusing is observed at the pulse's mesoscale. The refocused pulse is compared with the initial data used in the forward numerical experiment. Only a fraction of the total energy is refocused since we have a finite $t_{0}^{\prime}$.

4.3.2. Partial window experiment. Now we present an experiment exactly as before but with the cutoff function $G_{t_{0}^{\prime}}$ being the indicator function of $[0.25,0.5]$, that is $G_{t_{0}^{\prime}}=1_{\left[t_{0}^{\prime} / 2, t_{0}^{\prime}\right]}$. The forward experiment is exactly as before but we only timereversed the final half of the reflected wave. The same four stages of Figure 4.3 are repeated graphically for this partial window experiment (c.f. Figure 4.5). Refocusing still takes place but it is not as sharp as before. In particular there is more noise around the refocused pulse.

4.3.3. Medium-wave mismatch experiment. We repeat the (large window) time-reversal experiment relative to Figure 4.2 but with a different realization of the random medium. There is a mismatch between medium and wave because the reflected wave was not generated by this realization of the topography. As expected no refocusing takes place as can be seen in the bottom graph of Figure 4.6.

4.3.4. Self-averaging property. As mentioned in section 2.5 it can be shown that, in the limit $\varepsilon \downarrow 0$, the variance of $B_{0}^{T R}\left(t_{0}^{\prime}+\varepsilon t\right)$ goes to zero. This is the self-averaging property in the sense that the refocused profile is independent of the particular realization of the random medium. In order to show that the numerical code captures very well the self-averaging property we repeated the time-reversal 


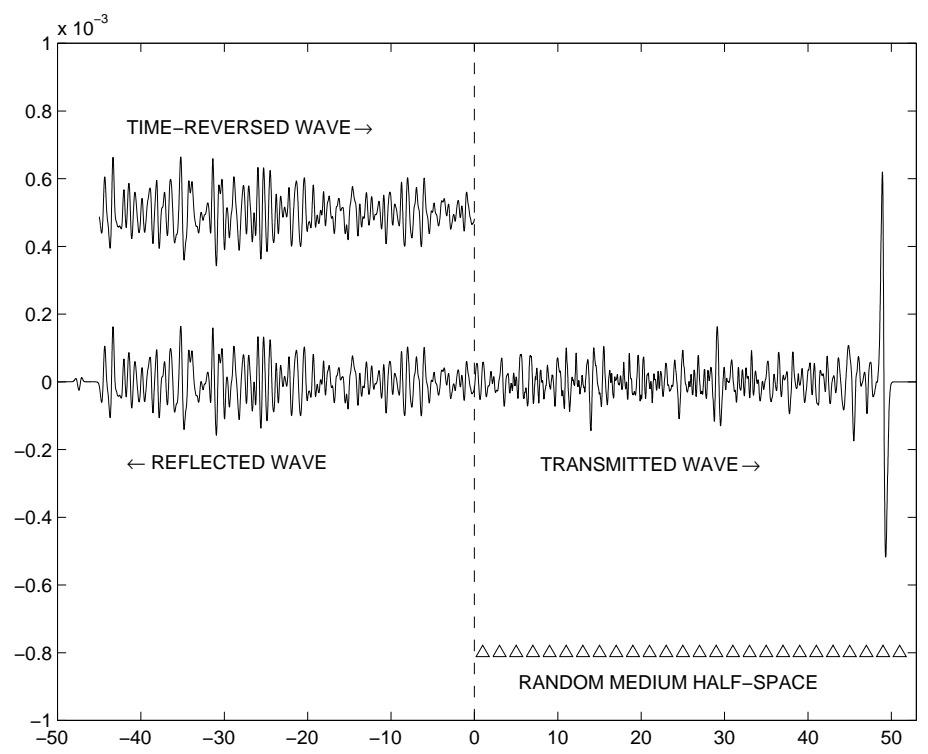

FIG. 4.2. The signal at the bottom shows the reflected wave on the left side of the origin and the wave propagating and interacting with the random medium on the right side of the origin, as shown in Figure 4.1. The top signal is the windowed reflected wave which will be time-reversed and sent back into the medium. Note that "what came out last will be sent back into the medium first".

experiment for 10 different realizations of the topography. All the parameters were kept fixed including the cutoff function $\left(G_{t_{0}^{\prime}} \equiv\right.$ indicator function of $\left.[0,0.5]\right)$. The shape of the refocused pulse is (statistically) very stable as can be seen in Figure 4.7. The fluctuations around each refocused pulse are not self-averaged quantities.

4.4. Weak nonlinear effect. This experiment is similar to the one presented in Figure 4.2. The topography is exactly the same. The only difference is that in the forward numerical experiment the amplitude-to-depth ratio has been increased by a factor of $10(\alpha=0.01)$. This weak nonlinear effect is enough to promote a mild steapening of the pulse front. This will correspond to the broadening of the pulse's Fourier spectrum. The higher frequencies generated interact more strongly with the random medium and therefore more reflection is observed (c.f. top of Figure 4.8).

The reflected wave is time-reversed as indicated by the windowed signal presented in the top graph of Figure 4.8. The cutoff function is the indicator function for [0, $0.5]$. The time-reversed wave is sent back into the random medium and refocusing takes place, as before, at $t=t_{0}^{\prime} / \varepsilon=50$. This result is presented at the bottom graph of Figure 4.8. From this numerical experiments, we have evidence that in this weak nonlinear regime the shape of the refocused pulse is not affected by the nonlinearity. At this time the nonlinear theory has not been developed.

\section{REFERENCES}

[1] M. Asch, W. Kohler, G. Papanicolaou, M. Postel, and B. White, Frequency content of randomly scattered signals, SIAM Review 33, (1991) 519-626.

[2] M. Asch, G. Papanicolaou, M. Postel, P. Sheng, and B. White, Frequency content of randomly scattered signals, Part I, Wave Motion 12, (1990) 429-450. 

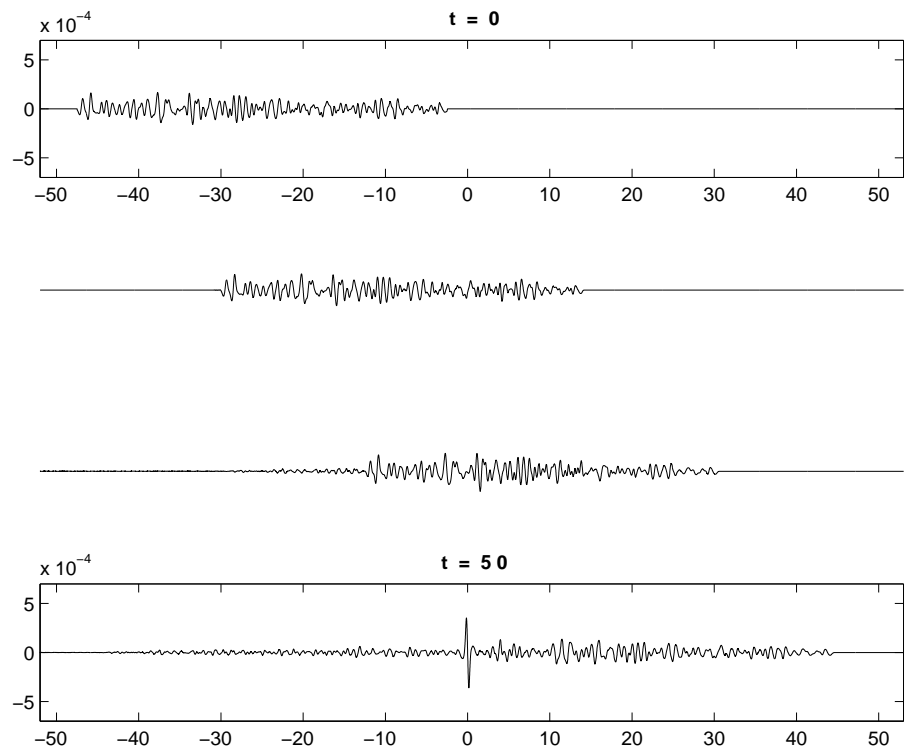

FIG. 4.3. The top graph shows the windowed wave sent back into the medium as obtained in Figure 4.2. The two intermediate graphs show the wave propagating into the medium. Observe that only a very small left going wave is generated (the "noisy" fluctuations visible at the left hand side of the third graph). The bottom graph shows the refocused pulse arriving at the origin at the predicted time.

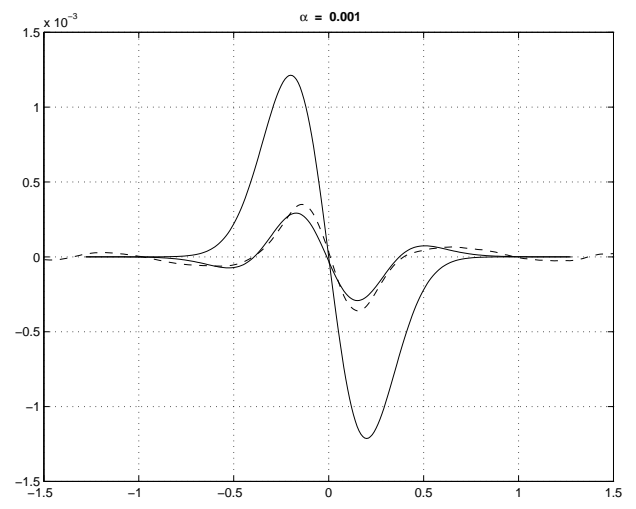

FIG. 4.4. The refocused pulses (solid line $=$ theory; dashed line $=$ numerical experiment) are compared with the initial pulse profile (large pulse; solid line). The pulse shape is fully recovered but the effect of the time-reversal window (i.e. the cutoff function $G_{t_{0}^{\prime}}$ ) is clearly observed as described in the equation (2.4).

[3] P. Blomgren, G. Papanicolaou, and H. Zhao, JASA, Vol 111, Super-Resolution in Time-Reversal Acoustics, Vol. 111, (2002), pp. 230-248.

[4] B. Burridge, G. Papanicolaou, P. Sheng, and B. White, Probing a random medium with a pulse, SIAM J. Appl. Math. 49, (1989) 582-607.

[5] B. Burridge, G. Papanicolaou, and B. White, Statistics for pulse reflexion from a randomly layered medium, SIAM J. Appl. Math., 47, (1987) 146-168.

[6] V. Casulli, and R.T. Cheng, Semi-implicit finite difference methods for three-dimensional shallow water flow, Int. J. Num. Meth. Fluids, vol. 15, (1982) pp. 629-648.

[7] J. Chillan and J.P. Fouque, Pressure fields generated by acoustical pulses propagating in ran- 

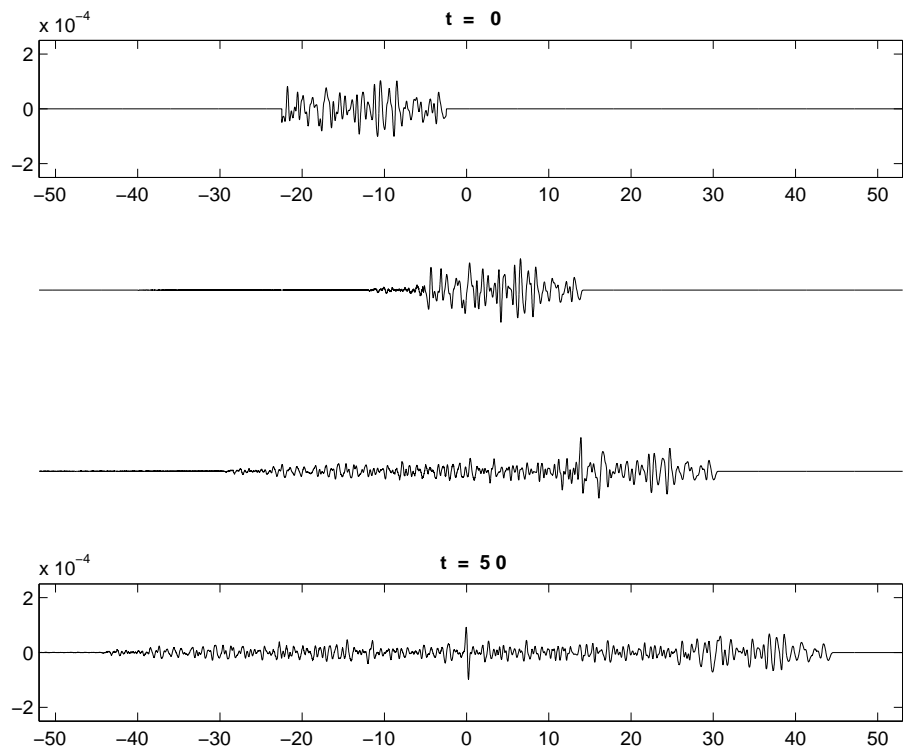

FIG. 4.5. This is a similar experiment as the one presented in Figure 4.3, but we have kept only the last half part of the reflected signal. We observe more noise aroud the refocused pulse.
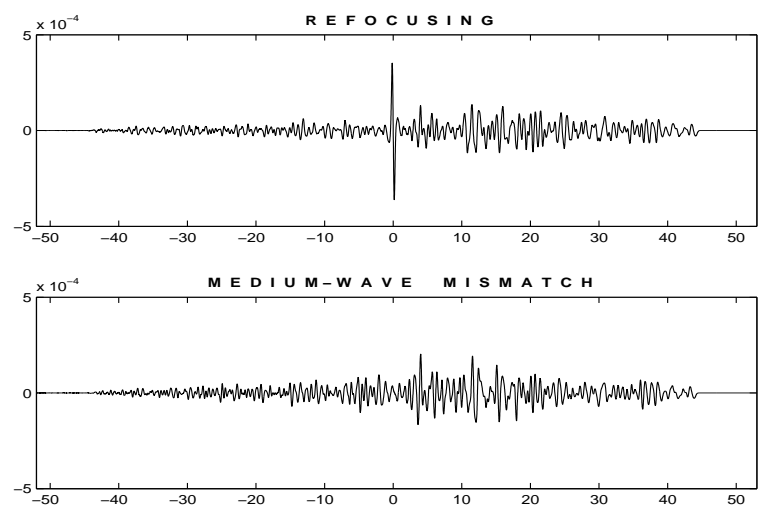

FIG. 4.6. In this experiment we send the time reversed signal in a different realization of the medium. The bottom graph shows the absence of refocusing compared to the top graph, which is identitical to Figure 4.3 at time $t=50$.

domly layered media, SIAM J. Appl. Math. 58, (1998) 1532-1546.

[8] J.F. Clouet and J.P. Fouque, Spreading of a pulse travelling in random media, Annals of Applied Probability 4, (1994) 1083-1097.

[9] J.F. Clouet, J.P. Fouque and M. Postel, Spectral analysis of ramdomly scattered signals using the wavelet transform, Wave Motion 22, (1995) pp. 145-170.

[10] J.F. Clouet and J.P. Fouque A time-reversal method for an acoustical pulse propagating in randomly layered media, Wave Motion 25, (1997) pp. 361-368.

[11] M. Fink Time reversal mirrors, J. Phys. D: Appl. Phys. 26, (1993) pp. 1333-1350.

[12] M. Fink, Time-reversed acoustics, Scientific American, November 1999, pp. 67-93.

[13] M. Fink and C. Prada, Eigenmodes of the time reversal operator: a solution to selective focusing in multiple-target media, Wave Motion 20, (1994) pp. 151-163.

[14] J.P. Fouque, J. Garnier and A. Nachbin, Time reversal for dispersive waves in random media, 


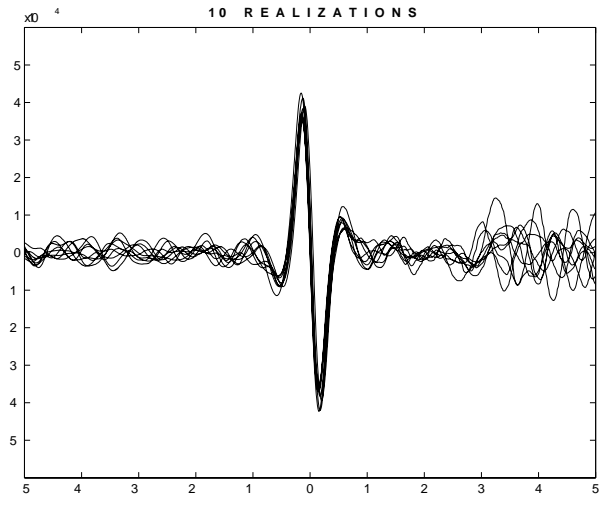

FIG. 4.7. We superimposed the refocused pulses obtained with ten independent realizations of the medium. Observe that the shape of the refocused pulse is independent of the realization as predicted by the asymptotic theory. The small fluctuations around are not self-averaged quantities.
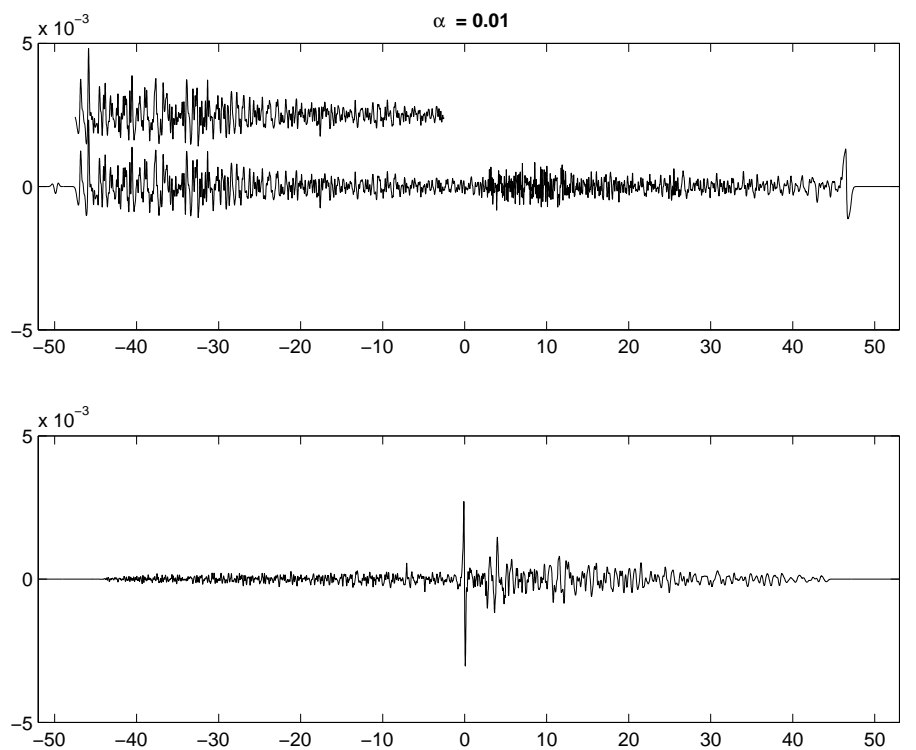

Fig. 4.8. This experiment is similar to the one presented in Figure 4.2. The only difference is that the pulse amplitude has been increased by a factor of 10, making the nonlinear term small but not negligible. We still observe refocusing in the bottom graph.

preprint, 2002.

[15] J.P. Fouque and K. Sølna, Beating the diffraction limit in laminated media, in preparation.

[16] J. Ndzie, Refocusing of a time-reversed acoustical pulse propagating in randomly layered media, J. Stat. Phys., Vol. 104, No. 516, (2001).

[17] W. Kohler, C. Papanicolaou, and B. White, Localization and Mode Conversion for Elastic Waves in Randomly Layered Media, Wave Motion 23, (1996) pp. 1-22 and pp. 181-201.

[18] P. Lewicki, R. Burridge and M. De Hoop, Beyond effective medium theory: pulse stabilization for multimode wave propagation in high-contrast layered media, SIAM J. Appl. Math. 56, (1996) pp. 256-276

[19] P. Lewicki, R. Burridge and G. Papanicolaou, Pulse stabilisation in a strongly heterogeneous layered medium, Wave Motion 20, (1994) pp. 177-195. 


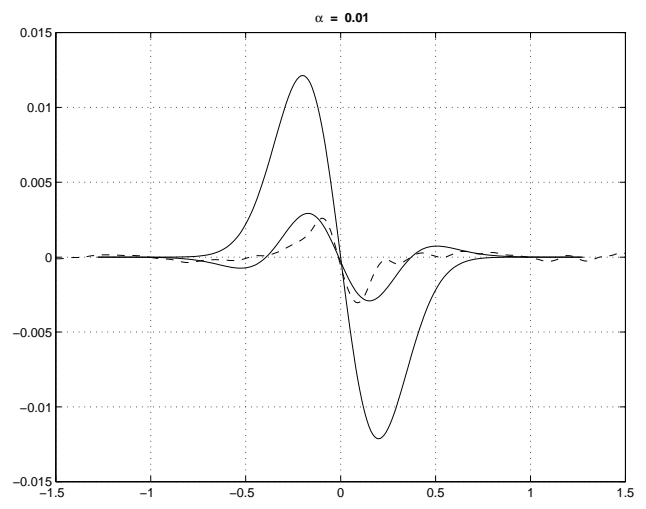

FIG. 4.9. The refocused pulses (solid line = linear theory; dashed line = numerical experiment) are compared with the initial pulse profile (large pulse; solid line). The pulse shape is fully recovered but the effect of the weak nonlinearity is starting to become noticeable.

[20] A. Nachbin and V. Casulli, Water waves: linear potential theory results validated with a hydrostatic Navier-Stokes model, In: Mathematical and Numerical Aspects of wave Propagation, Editor J. A. DeSanto, SIAM, (1998).

[21] R.F. O'Doherty and N.A. Anstey Refections on amplitudes, Geophysical Prospecting 19, (1971) pp. $430-458$.

[22] G. Papanicolaou, M. Postel, P. Sheng and B. White, Frequency content of randomly scattered signals, Part II, Wave Motion 12, (1990) pp. 527-549.

[23] G. Papanicolaou, L. Ryzhik and K. Sølna, Statistical stability in time reversal, (2002) submitted for publication.

[24] C. Pires and M.A. Miranda, Tsunami waveform inversion by adjoint methods, J. Geophys. Res., vol. 106, No. C9 (2001), pp. 19733-19796.

[25] K. Sølna, Stable spreading of acoustic pulses due to laminated microstructure, Ph.D. dissertation, (1997) Stanford University.

[26] G.B. Whitham Linear and nonlinear waves, John Wiley, (1974). 